\title{
Energy systems: a new look at aerobic metabolism in stressful exercise
}

\begin{abstract}
The generally accepted model of energy systems has been used for many years. When exercise intensity rises and glucose uptake by working muscles is increased, a drop in circulating glucose is observed while in higher intensity exercise, muscle glucose uptake can increase by as much as 30 - 50 times, compared to the resting rate. Changes is glucose metabolism have been described by numerous authors and forms an integral part of the modification of energy systems to training stimulus. This is integral to understanding the changes that may occur in the use of blood borne glucose and intra muscular glycogen that may be a marker of exercise intensity. Further Increased cytosolic and more effectively mitochondrial calcium during high ATP turnover in exercise increases glucose phosphorylation while in some cells MAS converted lactate to pyruvate whereas glucose oxidation and lactate production are influenced by the activity of MAS when the intensity of exercise rises, it can lead to down-regulation of MAS activity, which leads to an increase in lactate production Where MAS is inhibited, there is a $50 \%$ decrease in lactate oxidation. Likely sites for the adaptation of MAS are highlighted and the effect that this can have on our view of how the energy systems function during high intensity exercise are also suggested. Through consideration of these metabolic events a new vision of energy system function is proposed.
\end{abstract}

Keywords: energy systems, sports, lactate system
Volume 2 Issue I - 2018

\author{
Emma Swanwick, Martyn Matthews \\ School of Health Sciences, University of Salford, UK
}

Correspondence: Emma Swanwick, School of Health Sciences, University of Salford, UK, Email ek.swanwick@gmail.com

Received: December 06, 2017 | Published: January 19, 2018

\section{Introduction}

For many years the notion of the aerobic and anaerobic systems has been used to define the type of work being carried out by athletes during high intensity sports. The traditional view is that the resynthesis of ATP is supplied by three integrated systems comprising the Creatine Phosphate (PCr), lactic and aerobic systems. ${ }^{1}$ This now appears to have been incorrect. ${ }^{2}$ The three-system model does not explain why certain timelines of performance seem to exist in the aerobic area of metabolism. The balance of how each of these systems contributes at different times has been reviewed by a number of authors. As the anaerobic contribution declines, the aerobic increases with continued activity duration. ${ }^{3,4}$ Larsen (2010) referred to the first and second ventilatory thresholds, which in other papers have been defined as the aerobic threshold, lactate threshold, max-lass, anaerobic threshold and onset of Blood Lactate Accumulation (OBLA). ${ }^{5-7}$ These points have been seen as important in determining the transition from the predominance of the aerobic system to the "lactate system." This definition is incorrect however, as the thresholds speak more about the relationship between clearance rates and lactate production than lactate production alone. The literature is littered with confusion as to where each occurs and what it means, mostly due to the mix of protocols ${ }^{8}$ and subjects used (elite athletes, middle-aged sedentary, college students etc). The effect of this was to create confusion as to what went on, where and why. Although some activities may rely predominately on a single system (either very short or very long duration activities, where the percentage contribution from of the other systems is small), the timing and intensity of other activities require a significant contribution of more than one energy transfer system. All activities activate each energy system to some degree, depending on exercise intensity and duration.

During maximal efforts, the anaerobic (lactic) system lasts from 45 seconds to 2 minutes, after which all further exercise would be aerobic. ${ }^{9}$
In adults, maximal exercise of $60-120 \mathrm{sec}$ duration has resulted in attainment or near attainment of $\mathrm{VO}_{2}$ max. ${ }^{10-13}$ This illustrates that, if the intensity is high enough, there may be a significant contribution from the aerobic system and it may be possible to reach Peak Oxygen Uptake $\left(\mathrm{PVO}_{2}\right.$ ) in what are considered "anaerobic tests". ${ }^{11}$ As displayed in Figure $1,{ }^{14}$ the anaerobic reactions of glycolysis (shortterm energy transfer system) generate increasingly greater energy for ATP resynthesis when all-out exercise continues longer than a few seconds. An increase occurs in aerobic energy contribution very early in exercise but climbs at a slower rate than the anaerobic system delivery rate. ${ }^{15}$ Despite this, the energy requirement of all-out exercise significantly exceeds the energy generated by hydrogen's oxidation in the respiratory chain. This means that the anaerobic reactions of glycolysis predominate, with large quantities of lactic acid accumulating within the active muscle and ultimately appearing in the blood.

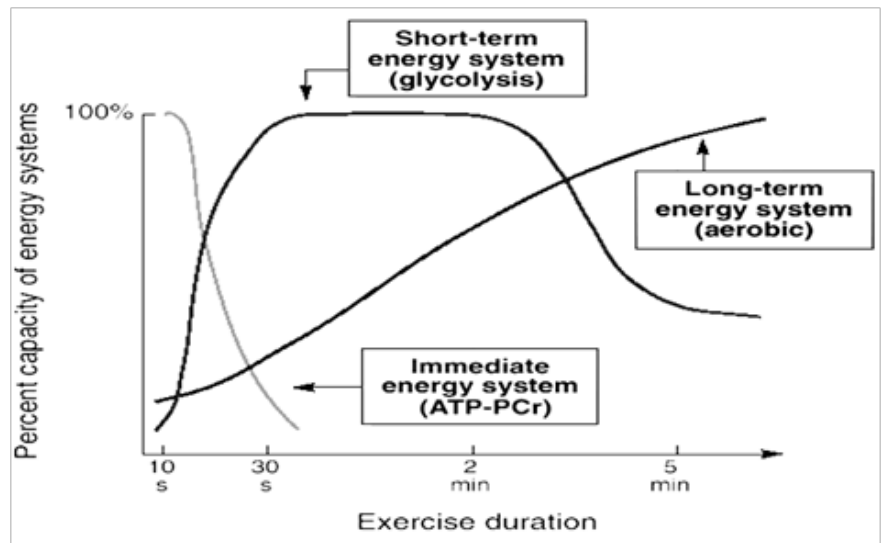

Figure I Shows the traditional model illustrating the involvement of anaerobic and aerobic energy transfer systems for different durations of allout exercise. ${ }^{14}$ 
Unlike tests for maximal oxygen uptake, no specific criteria exist to indicate that a person has reached a maximal anaerobic effort. Although motivation and the test environment likely influence the test score, researchers most commonly use the level of blood lactate to indicate the degree of activation of the short-term energy system. Activities requiring substantial activation of the short-term energy system demand maximal work for up to three minutes. All-out runs and cycling exercise have usually been used, although weight lifting of a certain percentage of maximum and shuttle runs are also used. ${ }^{16,17}$ Because age, sex, skill, motivation and body size affect maximal performance, selecting a suitable criterion test for developing standards for glycolytic energy capacity is difficult.

Figure $2^{18}$ presents the percentage contribution of each metabolic pathway during three different all-out cycle ergometer tests. The results are shown as a percentage of the total work output. Note the progressive change in the percentage contribution of each of the energy systems to the total work output as duration increases. Persons who engage in sports that require sustained, high-intensity exercise (i.e. endurance) generally possess a large aerobic energy transfer capacity. Men and women who compete in distance running, swimming, bicycling and cross-country skiing generally record the highest maximal oxygen uptakes. These athletes have almost twice the aerobic capacity of sedentary individuals. This does not mean that $\mathrm{VO}_{2} \max$ alone determines endurance exercise capacity. Other factors, especially those at the muscle level such as capillary density, enzymes and fibre type, strongly influence the capacity to sustain a high percentage of $\mathrm{VO}_{2}$ max. However, the $\mathrm{VO}_{2} \max$ does provide useful information about the capacity of the long-term energy system. For example, attainment of $\mathrm{VO}_{2}$ max requires integration of ventilatory, cardiovascular and neuromuscular systems; this gives significant physiologic "meaning" to this metabolic measure. For these reasons, $\mathrm{VO}_{2}$ max represents a fundamental measure in exercise physiology and often serves as the standard against which to compare performance estimates of aerobic capacity and endurance fitness. Because it is the aim of training to induce an adaptive response that is both useful to the overall development of an athlete and that will ultimately transfer to better performance, it is crucial to understand both the adaptive mechanisms that cause the benefits and the intensity and duration of activity to optimally stimulate these. The intensity at which these training induced modifications take place is still open to much conjecture in science, or the proverbial "thumb suck" or art of coaching.

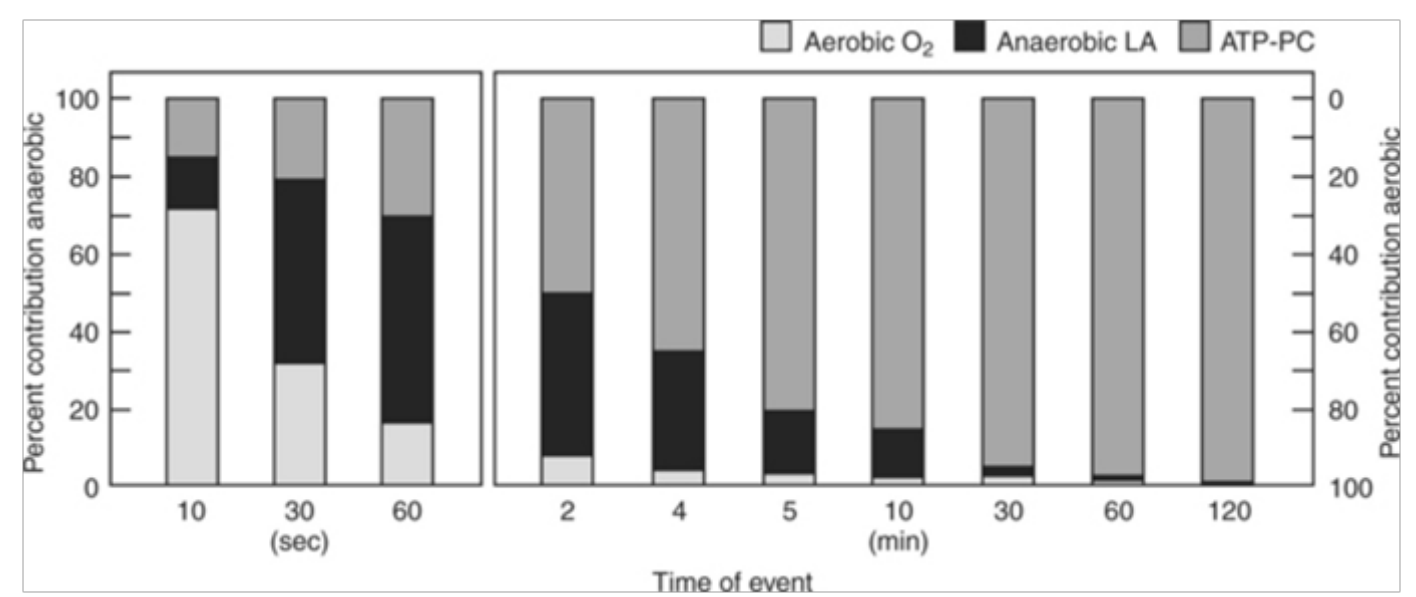

Figure 2 Time energy system continuum. Approximate relative contributions of aerobic and anaerobic energy production at maximal maintainable intensity for varying durations. The graphs assume $100 \%$ maximal oxygen uptake $\left(\mathrm{O}_{2}\right.$ max) at 10 minutes; $95 \% \mathrm{O}_{2}$ max at 30 minutes; $85 \%$ of $\mathrm{O}_{2}$ max at 60 minutes; and $80 \%$ $\mathrm{O}_{2}$ max at 120 minutes. Adenosine triphosphate-phosphocreatine $\leq 10$ seconds. ${ }^{18}$

There is, then, a need for some clearly definable markers of intensity that represent the key metabolic points at which athletes can train to increase the likelihood of the targeted adaptation. The use of the ventilatory thresholds, heart rate values, ${ }^{15}$ Conconi test, lactate thresholds etc has brought about much work with varying results, some good, ${ }^{19}$ some less so. ${ }^{8}$ There is generally a lack of literature that pinpoints the timelines for working at various intensities and how over- or under- working at these may affect the outcome of the training being carried out. There is also little explanation as to why certain thresholds appear to take place when they do, or rather why they occur within quite such tightly defined timelines. In this sense, the usefulness of the traditional aerobic/anaerobic model is limited.

\section{Our proposed hypothesis: 4 parts to the system of energy delivery}

At this point, along with the three previously defined "Systems", we suggest a change to the terminology. The use of glycolysis and glycogenolysis are mostly referred to in tandem during the aerobic/ anaerobic processes. This may be appropriate in lower intensity work where, due to fibre cycling, there is a combined use where glycogenolysis contributes to the glycolysis process without affecting the overall balance of muscle glycogen stores due to recycling. In higher intensity activity, however, we suggest that these two processes need to be considered separately in terms of exercise metabolism and muscle fibre recruitment. This is the basis for the four-system model, discussed below. The following describes these events described above into a logical order of increasing intensity (Table 1).

Table I Proposed definition of an adapted energy supply at a range of percentages of Maximal Oxygen Uptake $\left(\mathrm{MVO}_{2}\right)$

\begin{tabular}{lll}
\hline $\begin{array}{l}\text { Low intensity } \\
\text { work }\end{array}$ & $\begin{array}{l}\text { Below } 65 \% \\
\text { MVO2 }\end{array}$ & $\begin{array}{l}\text { Predominantly fatty acid \& some } \\
\text { blood sugar }\end{array}$ \\
$\begin{array}{l}\text { Moderate } \\
\text { intensity work }\end{array}$ & $\begin{array}{l}65 \% \text { to } 80 \% \\
\text { MVO2 }\end{array}$ & $\begin{array}{l}\text { Predominantly Aerobic Glycolysis } \\
\text { (from blood glucose) }\end{array}$ \\
$\begin{array}{l}\text { High intensity } \\
\text { work }\end{array}$ & $\begin{array}{l}80 \% \text { to } 100 \% \\
\text { MVO2 }\end{array}$ & Malate Aspartate shuttle \\
\hline
\end{tabular}


Our rationale for this thought process is as follows. Substrate utilization during exercise is determined by the intensity and duration of that exercise, as well as by the training status of the athlete and the availability of substrates. ${ }^{20}$ At rest and at low intensities (below 50\% $\mathrm{MVO}_{2}$ ), fat oxidation is generally the major energy source. ${ }^{21}$ Above this intensity, oxidation of glucose appears to become more important until it becomes the dominant fuel during high intensity aerobic work and potentially the only fuel source during anaerobic work.

\section{The rationale and supporting evidence for the model}

During higher intensity exercise, muscle glucose uptake can increase by as much as $30-50$ times, compared to resting rate. ${ }^{20}$ This also generally corresponds to the output of glucose from the liver. Exercise can induce the uptake of glucose, which is independent of insulin action and which, under certain conditions, can outstrip the rate of utilization..$^{22,23}$ The rate of glucose uptake (Rd) during exercise does not demonstrate a simple linear relationship with oxygen uptake. During low intensity exercise, there appears to be a small increase in the rate of disappearance (Rd) but this does not line up with the observed 4-fold increase in $\mathrm{CO}_{2}$ production or $\mathrm{VO}_{2}{ }^{24}$ The suggested down-regulation of mitochondrial fatty acid uptake in conditions created by higher intensity exercise ${ }^{21}$ may be a function of oxygen use. A two-carbon molecule produced from beta-oxidation takes more oxygen to process than the one-carbon units produced from glycolysis and this is regarded as one of the controlling factors in the switch from fatty acid oxidation to glucose oxidation with the increase in exercise intensity. ${ }^{21}$

\section{Glucose and glycogen usage at different intensities}

Experiments by Greenhaff et al. ${ }^{25}$ have shown that when there is enough time for oxidative phosphorylation to maintain the ATP resynthesis in type I fibers, activation of glycogenolysis does not take place in these type of fibre. When activity rate is increased however, glycogenolysis occurs at its maximal rate. The rate of glycogenolysis in fast twitch fibers, as determined by the amount of phosphorylase available in the muscle fibre, was also found to take place at its maximal rate. From this we might conclude that the overall rate of glycolytic activity in a muscle and therefore the degree to which the appearance of the metabolites of anaerobic glycolysis and glycogenolysis, is dependent firstly on the amount of time between the contractions required and then the number of fast twitch fibers that are being recruited. Epinephrine infusion was found to stimulate greater glycolytic activity in slow twitch fibers but not fast twitch fibers and this may suggest a relationship to the increase in circulating AMP which activated the phosporylase activity.

Margaria et al. ${ }^{26}$ first put forward the model that when phosphocreatine stores were depleted, this would stimulate the glycolytic activity to replenish ATP. This view was challenged by Bergstrom and developed further by Boobis et al. ${ }^{27}$ who found that contrary to what Margaria had postulated, glycolytic activity is stimulated at the start of exercise. It is also now accepted that the momentary rise in ADP concentration, created at the initiation of muscular contraction, is enough to stimulate the onset of phosphocreatine $(\mathrm{PCr})$ hydrolysis and, most probably, is the primer for anaerobic glycolysis to increase its activity also. Depletion of $\mathrm{PCr}$ leads to a rapid reduction in energy derived from this source (within 10 seconds PCr activity is less than $50 \%$ of its initial activity). Resynthesis of $\mathrm{PCr}$ is also reliant upon oxidative phosphorylation, ${ }^{28}$ which can often only take place at the end of exercise or when the intensity is reduced sufficiently to allow energy derived by this mechanism to be re-directed from predominantly ATP synthesis to $\mathrm{PCr}$ resynthesis.

The initiation of muscular contraction requires the release of $\mathrm{Ca}^{2+}$, which is a stimulant for glycogenolysis and glycolysis. Additionally, the products of ATP hydrolysis and PCr hydrolysis (ADP, AMP, IMP, $\mathrm{NH} 3$ and inorganic phosphate (Pi)) also act as stimulants of glycolysis and glycogenolysis. Although IMP is thought to exert its effect upon phosphorylase $\mathrm{b}$ (which brings about glycogenolysis), because of their close relationship with ATP turnover, AMP and Pi concentrations are considered to be the key regulators of glycogen degradation during muscle contraction. ${ }^{20}$ It is already understood that as exercise begins there is an initial increase in the production of glucose by the liver. In moderate to high intensity work, the raised glucose output is maintained by an accelerated liver glycogenolysis (demonstrated by reduced liver glycogen). ${ }^{29}$ This production of hepatic glucose is carefully monitored and is sensitive to feedback signals within the system. This is demonstrated by glucose infusion only results in a moderate change in plasma glucose.$^{30}$ It is important at this point to not mix up hepatic glycogenolysis with muscle glycogenolysis.

As much as $85 \%$ of the change in glucose release and uptake by the cell is controlled by mechanisms other than insulin response. ${ }^{29}$ Insulin levels are reduced during exercise while at the same time, the uptake of glucose may increase. This suggests that other mechanisms are at work in the control of glucose activity during exercise. Glucose transporters in the cell membrane (GLUT4 specifically) do become more sensitive to insulin during exercise. It has also been demonstrated that muscle contractions stimulate the activity of GLUT4 transporters from a different pool and that the two mechanisms (contraction and insulin effect) are additive. ${ }^{31}$ Insulin level increases with training. That is, a person who demonstrates greater aerobic fitness will have a higher level of circulatory insulin than a less aerobically trained athlete. ${ }^{32}$ This is a reasonable response, as a raised insulin level will reduce the amount of glucose released from the liver to the blood. Although the increased insulin would be expected to raise glucose uptake into the working cells, this may only account for $15 \%$ of glucose movement. ${ }^{29}$

When exercise intensity rises and glucose uptake by working muscles is increased, a drop in circulating glucose is observed.$^{33} \mathrm{At}$ the start of exercise or in intense exercise there is a pronounced hormonal response (epinephrine), which is stimulated by the degree change in the central mechanisms and an increase in central motor activity. This drives glucose mobilization that will exceed the peripheral glucose uptake. ${ }^{24}$ The result of this is an observable rise in blood glucose. The mechanisms that drive this response are not fully understood. It is known that the sympathetic liver innervations has little if any role in hepatic glucose production during exercise and that cortisol and growth hormone only play a minimal role in inducing the rise in glucose output. ${ }^{34}$ Hormonal mechanisms, however, are seen to only partially explain the stimulation of the production of glucose during exercise. Investigation of a wider identification of factors, which may stimulate the rise in glucose production from the liver during exercise and intense exercise in particular, is needed. ${ }^{35}$

\section{Blood glucose responses to increased workloads}

Most of the studies investigating the blood glucose response to exercise have tested during moderate intensity or endurance based exercise. ${ }^{36-38}$ The effects of higher intensity exercise however produce a different set of circumstances for cellular respiration. The demand 
for energy is greater and the requirement to use anaerobic metabolism (glycolytic metabolism) will create a much greater demand for glucose within the working cell. Fujitani looked at the long-term effects of strenuous resistance training on glucose use. The findings were that this kind of training increased glucose use and the sensitivity to insulin also. This suggests that athletes who do more high intensity work will develop a greater sensitivity to glucose and will potentially use more glucose per unit of work done. The effect of this on the crossover from fatty acid oxidation to glucose oxidation may reduce the reliance on fatty acid oxidation.

Coggan ${ }^{39}$ and coworkers looked at the effects on glucose kinetics in subjects with different lactate thresholds and found that the rate of glucose appearance and utilization was lower in subjects with high lactate thresholds compared to those with lower lactate thresholds. This would suggest that there is a difference in muscle respiratory rate in such comparisons. As such, observation of the changes in glucose and lactate kinetics would provide a view of the changes in respiratory rate, which would not be possible from lactate alone. In both groups, the relative oxygen consumption was the same, however the rate of glucose utilization was $17 \%$ lower in the high lactate threshold subjects. This would have a marked effect upon the endurance capacity of the subjects, on the efficiency of the subjects' metabolism and potentially on the anaerobic capacity of the subjects also.

$\operatorname{Coggan}^{39}$ noted that with untrained humans working at higher intensities $\left(80 \%\right.$ of $\left.\mathrm{MVO}_{2}\right)$ there was a decrease in insulin clearance allowing its concentration to actually increase, however neither this nor increases in glucagon played a part in the observed rise in plasma glucose associated with the workload. Coggan ${ }^{39}$ has suggested that training, even at this relatively high intensity, brought about a reduction in the appearance and utilization of plasma glucose. He surmised that this was due to a reduction in muscle glucose transport and that this was related to a training-induced increase in muscle mitochondrial respiratory capacity. The reduction in glucose production and utilization was seen as a possible factor in increasing endurance seen with training.

In agreement with Fujitani \& Brooks ${ }^{32}$ suggested that at higher intensities the rate of glucose uptake is actually increased. Certainly the higher intensities of exercise studied $\left(80 \%\right.$ of $\left.\mathrm{MVO}_{2}\right)$ are, for many athletes, below the lactate threshold and as such could still be seen as predominantly aerobic in nature. This intensity may also elicit some fatty acid oxidation and be below the point where glucose utilization is sufficient to cause a marked increase in the rate of appearance and oxidation. Malate Aspartate and Glycerol phosphate shuttle; overlooked importance to control of exercise intensity.

Mitochondria of glycolytic muscle fibers are well adapted to play a central role for maintaining a satisfactory redox state in these fibers. ${ }^{40}$ An elevation of blood glucose accelerates mitochondrial oxidative phosphorylation through NADH transfer via the Malate aspartate shuttle (MAS) and $\mathrm{FADH}_{2}$ through the Glycerol-3-phosphate shuttle (G3PS). ${ }^{41}$ What mechanism controls this and how it interacts with relative exercise intensity has not been considered.

Zhou observed that the rate of glycogen breakdown is a major determinant of cytosolic NADH/NAD and lactate production, however it did not affect mitochondrial metabolism or steady state lactate production. Increased shuttle activity is known to lower cytosolic NADH significantly and can be seen as a key controller of lactate production both during ischemia and steady state. Blanchaer et al. ${ }^{42}$ found that $\alpha$-glycerol-phosphate de-hydrogenase activity in white muscle fibers was more than twice that of red muscle fibers. This gives us an indication that the glycerol-phosphate shuttle does actually have wider roles to play in muscle metabolism than previously thought and is not just limited to Esterification. It may have an important part to play in metabolism that would affect moderately high intensity exercise.

MacDonald \& Brown ${ }^{43}$ provided an interesting observation in that key enzymes of the G3PS were found to contain calcium-binding sequences that explained the calcium activation of the enzyme and that this might also fluctuate sufficiently to allow G3PS to participate in glycolytic functions. Its role in skeletal muscle was further elaborated by MacDonald \& Marshall ${ }^{44}$ who showed that a nonfunctioning G3PS caused a block in glycolysis at the step catalyzed by glyceralderhyde phosphate dehydrogenase and severely affected the action of skeletal muscle. Increased in cytosolic and more effectively mitochondrial calcium during high ATP turnover in exercise increases glucose phosphorylation. ${ }^{41}$ Contreras et $a .^{45}$ argued $\mathrm{Ca}^{2+}$ control of MAS action in a range of tissues, including skeletal muscle, may demonstrate tissue specific protein forms as yet un defined that do allow the rise in respiration do exist. MAS only require minute changes of $\mathrm{Ca}^{2+}$ to create an up-regulation and prolong mitochondrial energization. It has also been suggested that MAS plays a major role in increasing the metabolic fitness of the cell. ${ }^{46}$

Observation of the action of the (MAS) in brain tissue ${ }^{47}$ found that Lactate Dehydrogenase acted with MAS to greatly increase respiration rate and use of oxygen. The acceleration of the MAS also needed sufficiently high levels of $\mathrm{Ca}^{2+}$ to reach maximum efficacy. The action of MAS allowed a strong up-regulation of the supply of pyruvate to the mitochondria. However, when $\mathrm{Ca}^{2+}$ rate of appearance gets too high, the concentration of this $\mathrm{Ca}^{2+}$ within the mitochondria may also rise. Large $\mathrm{Ca}^{2+}$ loads are initially buffered by fast mitochondrial sequestration that effectively uncouples electron transport from ATP synthesis, leading to an increase in $\left(\mathrm{H}^{+}\right) .{ }^{48}$ If this continues long enough, an increased net influx of calcium into cells triggers a "vicious cycle" of mitochondrial calcium overloading and energy depletion. ${ }^{49}$

The function of MAS also requires glutamate. Without this, no up-regulation can occur. With it and in the right conditions, $1 \mathrm{NADH}$ is generated in the mitochondria. When MAS is fully up-regulated, 1 pyruvate molecule can also be supplied into the mitochondria allowing creation of $5 \mathrm{NADH} / \mathrm{FADH}_{2}$ molecules. An electro-chemical pump augments mitochondrial uptake of NADH and Pyruvate. This then acts like an overdrive mechanism of mitochondrial respiration. Pellerin also noted that in some cells MAS converted lactate to pyruvate whereas glucose oxidation and lactate production are influenced by the activity of $\mathrm{MAS}^{50}$ and requires oxygen to function.

The increase in Lactate dehydrogenase activity (and the production of lactate) is the compensatory action for MAS to keep pace with cytosolic $\mathrm{NAD}^{+}$demand as proposed by Shantz. ${ }^{51}$ This lead $\mathrm{Kane}^{52}$ asked the question "why should lactate be produced under fully aerobic conditions, as all pyruvate should be going through the mitochondria for oxidative phosphorylation and the MAS should be regenerating sufficient $\mathrm{NAD}^{+"}$. The primary regulator of pyruvate oxidation through the pyruvate dehydrogenase complex (PDC) is mitochondrial NADH/NAD. ${ }^{53,54}$ Elustondo and Jacobs both found that lactate was oxidized in the presence of $\mathrm{NAD}^{+}$, malate and ADP. 
Allowing pyruvate to be transported into the mitochondria, this would provide an important pathway for control of $\mathrm{pH}$, cycling of lactate and allowing continued aerobic metabolism when the PDH route is blocked into Krebs cycle that should lead to onset of anaerobic metabolism. Lu et al. ${ }^{55}$ showed that when the intensity of exercise rises, it can lead to down-regulation of MAS activity, which leads to an increase in lactate production Where MAS is inhibited, there is a $50 \%$ decrease in lactate oxidation in synaposomes and this may be created by MAS competing with $\alpha$-ketoglutarate dehydrogenase for a shared substrate, $\alpha$-ketoglutarate. This then becomes a rate limiting effect on MAS to shuttle NADH into the mitochondria.

MAS have a far greater role to play in retaining the equilibrium within skeletal muscle that allows higher intensity of aerobic exercise to continue in less aerobically friendly conditions. Kane ${ }^{52}$ suggested that the link between lactate \& MAS allows the transfer of a proton from lactate to the inner mitochondrial membrane. By doing so, both $\mathrm{pH}$ balances can be retained and greater pyruvate can also enter the mitochondria while also regenerating $\mathrm{NAD}^{+}$. In glycolysis, 2 pairs of hydrogen ions are stripped from glucose and their electrons being passed to NAD to form NADH. Unlike heart, liver and kidney cells, muscle cells remain impermeable to NADH. In this situation, electrons from extra-mitochondrial NADH are shuttled indirectly into the mitochondria via MAS. This ends with the electrons being passed to FAD to form $\mathrm{FADH}_{2}$ and enters the electron transport chain at the second step of ATP formation. This produces a total of 4 ATP molecules (net of 6 ATP) by aerobically generated oxidative phosphorylation in skeletal muscle. With the addition of a pyruvate molecule being passed through to the mitochondria a further $5 \mathrm{NADH} / \mathrm{FADH}$ can be created (between $7 \& 10$ ATP). As such this mechanism would sustain aerobic metabolism significantly, particularly when under aerobic stress. Indeed, the timeline that would be produced by this smaller generation of ATP per unit of oxygen would probably sustain activity for approx. 20-30 mins where $500 \mathrm{~g}$ of glycogen was available in the skeletal muscles. ${ }^{56}$

Shantz et al. ${ }^{51}$ looked at what the effect of endurance training was on the Malate-aspartate and a-glycerol-phosphate shuttles and found that in the trained state, MAS activity increased by approximately $50 \%$. G3PS activity did not significantly change. If the findings of Blanchaers ${ }^{42}$ are correct, then this is no surprise, as the endurance activity would not have created sufficient loading to stimulate "white fiber" activity. Combined with the doubled capillary to fiber ratios shown to occur with endurance type stimulation ${ }^{5}$ and other intramitochondrial enzyme increases known to occur with such training, then endurance training can be seen to markedly increase oxidative capacity with action through the glycolytic pathway and glycolytic fibers.

\section{Selective muscle fibre type using different systems at the same time}

Human muscle fibre types are divided into 3 main types, Slow Twitch (STw), Fast Twitch A (FTa) and Fast Twitch x (FTx). ${ }^{57}$ Stimulation of the recruitment of muscle fibers follows a ramping process as described by Willmore \& Costill $^{58}$ and the increase in FTa fibers is signalled by either a reduction in glycogen in STw fibers or an increase in demand for muscle tension by the power demand of the work undertaken. At higher intensities FTx are also recruited.

Both Wilmore et al..$^{55}$ and Brooks ${ }^{36}$ have suggested that training stimulus is able to create adaptations in muscle fibers activity so that STw can become more like FTa and the same work would also see a reduction in FTx percentage as adaptations also increase the prevalence of FTa fibre ability. This increases the buffering capability and lactate removal from the FTa cells. It would seem sensible to also suggest that the metabolic changes identified in this paper would also occur in these FTa cells. This would increase the capacity at which high power could be maintained at the expense of the longer duration of STw and the higher power created by training FTx characteristics.

\section{What does this mean in an exercising situation?}

Stainsby et al. ${ }^{59}$ suggested that the formation of lactate was caused by a mismatch between glycogenolysis, glycolysis and pyruvate dehydrogenase complex (PDC) activation. Putman et al. ${ }^{6}$ demonstrated that PDC also has a major role to play in the accumulation of lactate in repeated bouts of exercise. Linnarsson et al. ${ }^{61}$ suggested that the buildup in oxygen deficit is due to a lag in oxygen utilization rather than oxygen delivery. Williamson et al. ${ }^{62}$ supported this premise when they demonstrated that lowering blood flow does not change the oxygen uptake kinetics at the onset of exercise. This is also important for recognizing that observations under hypoxic conditions, that change the time course of pulmonary oxygen uptake and increases oxygen deficit and $\mathrm{PCr}$ degradation, are different from the mechanism that creates the deficit in high intensity exercise. Further to this, Sahlin et al. ${ }^{20}$ also demonstrated that $\mathrm{PCr}$ degradation rate was not changed by the rate of oxygen delivery and concluded that it must be independent of oxygen availability. Timmons et al ${ }^{61}$ showed that lactate formation was largely unrelated to oxygen availability and with this in mind it is more likely that lactate accumulation reflects the reduction in the rate of cellular phosphorylation.

The separation of aerobic glycolysis and glycogenolysis is a demarcation line of relative intensity. It is also dependent upon the pattern of muscle fibre recruitment. Glycogenolysis only occurs in slow twitch fibers due to a high demand for energy through muscle tension. It is an all or nothing response, so glycogenolysis occurs at maximal level or not at all. Similarly, when fast twitch fibers are recruited, they predominantly use glycogenolysis which is either all or nothing in its function.

In this model the glycerol phosphate shuttle has more functionality once glycogenolysis comes into play. The generation of ATP through this pathway produces a net of 7ATP

(1 ATP saved from the use of glycogen, net of 2 produced via glycolysis and 4ATP produced by reduction of NADH to FADH/FAD through to $2^{\text {nd }}$ step of the electron transport chain).

This model also demonstrates how the increase in lactate can be controlled while reducing the oxygen requirement. The use of this pathway can be maintained for a longer period of time, dependent upon the rate of work being carried out. This is seen in the reduction of the Oxygen fraction extraction that takes place as intensity rises from the ventilatory threshold. The increase in glycogenolysis creates a higher level of glucose-6-phosphate within the cell, which blocks the further uptake of plasma glucose. This is not the case in all cells; rather it is a function of the number and type of muscle cells recruited. It has also been noted that the rise in plasma glucose is not linear with oxygen uptake (REFS). The rise in plasma glucose noted in our studies, could therefore be a marker of the onset of glycogenolysis.

This model therefore will split the function of the aerobic system into two, that which produces 36ATP from plasma glucose, which is carried through aerobic glycolysis and that which can produce 7ATP 
from aerobic glycogenolysis. The recruitment of the relative muscle fibers will then give a mixture of these two pathways. This would be why, with relative improvements in efficiency, the energy cost of an exercise can be reduced (Figure 3) (Table 2).

Table 2 Four energy system model and their percentage contribution to total energy output during all-out exercise of different durations. ${ }^{33}$

\begin{tabular}{llllllllllll}
\hline Sec & 5 & $\mathbf{2 4}$ & $\mathbf{4 8}$ & $\mathbf{1 2 0}$ & $\mathbf{2 4 0}$ & $\mathbf{4 2 0}$ & $\mathbf{8 4 0}$ & $\mathbf{1 8 0 0}$ & $\mathbf{3 6 0 0}$ & $\mathbf{5 4 0 0}$ & $\mathbf{7 2 0 0}$ \\
\hline CP & 80 & 20 & 11 & 7 & 4 & 2 & 1.5 & $\mathrm{I}$ & $\mathrm{I}$ & 0.5 & 0 \\
Anaerobic & 12 & 65 & 60 & 41 & 14 & 5 & 4 & 3 & 2 & 1 & 0 \\
Aerobic & 3 & 5 & 10 & 19 & 24 & 28 & 34 & 44 & 65 & 85 & 100 \\
Glyco & 5 & 10 & 19 & 33 & 51 & 65 & 61 & 52 & 32 & 14 & 0 \\
\hline
\end{tabular}

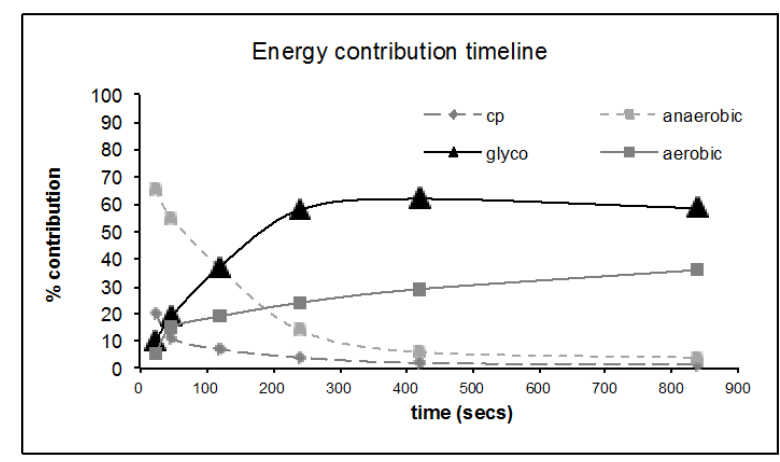

Figure 3 Schematic of the proposed 4 pathway energy time line. ${ }^{33}$ Four energy system model and their percentage contribution ( $Y$-axis) to total energy output during all-out exercise of different durations ( $\mathrm{X}$-axis).

\section{Conclusion}

Much research is based on sub maximal intensity and submaximal effort but these results are then used to extrapolate what is expected at higher intensity levels. Actual measurement of world class performance is scant as it is usually deemed to be too difficult to achieve accurate results. We collected data on national and international level swimming performances immediately following completion of race events and used this to model the responses seen in this paper. A model of three energy pathways, CP, Anaerobic and Aerobic has been used for a number of years. It has given overall definition to the use of energy systems but has not allowed many of the distinguishing points of exercise limitations to be explained. A new model has been proposed based upon the recruitment of muscle fibre type and the difference in glycolysis and glycogenolysis. This adds a 4th element to the pathway model. By doing this we are able to accurately predict timelines to exhaustion in performance level sporting activities.

This new model of explaining the time line of pathways at maximal effort, over both short time periods $(20 \mathrm{sec})$ and long time periods (up to 60 minutes), has proved to be accurate in identifying what has taken place within the race performance. Using data from this study, it has been possible to create a predictive tool that allows us to observe the individual response to high demand sporting events that matches the observations within the race. This provides an accurate tool for assessing training outcome and suitability to ensure that the athlete is correctly prepared for their target events at target competitions. ${ }^{62-72}$

\section{Acknowledgements}

None.

\section{Conflict of interest}

Author declares that there is no conflict of interest.

\section{References}

1. Robergs RA, Roberts SO. Exercise Physiology: Exercise, performance and clinical applications. Mosby, USA; 1996. p. 736.

2. Gastin PB. Energy System Interaction and Relative Contribution during Maximal Exercise. Sports Med. 2001;31(10):725-741.

3. Duffield R, Dawson B, Goodman C. Energy system contribution to 100$\mathrm{m}$ and 200-m track running events. $J$ Sci Med Sport. 2004;7(3):302-313.

4. Duffield R, Dawson B, Goodman C. Energy system contribution to 400-metre and 800-metre track running. $J$ Sports Sci. 2005;23(3):299-307.

5. Reichmann H, Hoppeler H, Costello MO, et al. Biochemical and ultrastructural changes of skeletal muscle mitochondria after chronic electrical stimulation in rabbits. Pflügers Archiv. 1985;404(1):1-9.

6. Pedersen PK, Juel C. Plasma acid-base status and hyperventilation during cycling at MAXLASS in low and high lactate responders. Medicine \& Science in Sports \& Exercise. 2001;33(5): S314.

7. Gladden LB. Lactate metabolism during exercise. Principles of Exercise Biochemistry. 2004;46:152-196.

8. Rushall B. USRPT and Training Theory VI: The Individuality Principle. Swimming Science Bulletin, USA; 2017.

9. Medbø JI, Mohn AC, Tabata I, et al. Anaerobic capacity determined by max accumulated Oxygen deficit. J Appl Physiol. 1988;64(1):50-60.

10. Astrand PO, Saltin B. Oxygen uptake during the first minutes of heavy muscular exercise. J Appl Physiol. 1961;16:971-976.

11. Gastin PB, Lawson DL. Variable resistance all-out test to generate accumulated oxygen deficit and predict anaerobic capacity. Eur J Appl Physiol Occup Physiol. 1994;69(4):331-336.

12. Katch V. Kinetics of oxygen uptake and recovery for supramaximal work of short duration. Int Z Angew Physiol. 1973;31:197-207.

13. Kavanagh MF, Jacobs I. Breath-by-breath oxygen consumption during performance of the Wingate test. Can J Sport Sci. 1988;13(1):91-93.

14. Powers SK, Howley ET. Exercise Physiology, Theory and application to physical performance, 7 th edn. McGraw Hill int, USA; 2009. p. 440 .

15. Ardle WD, Katch FI, Katch VL. Essentials of Exercise Physiology. Lippincott Williams \& Wilkins, USA; 2006. p. 290-294.

16. Stølen T, Chamari K, Castagna C, et al. Physiology of soccer: an update. Sports Med; 2005;35(6):501-536. 
17. Wisløff U, Castagna C, Helgerud J, et al. Strong correlation of maximal squat strength with sprint performance and vertical jump height in elite soccer players. Br J Sports Med. 2004;38(3):285-288.

18. Plowman SA, Smith DL. Exercise Physiology for Health. Fitness and Performance, 2nd ed. Benjamin Cummings, USA; 2003.

19. Matsunami M, Taguchi M, Taimura A, et al. Relationship among different performance tests to estimate maximal aerobic swimming speed. Medicine and Science in Sports and Exercise. 1999;31(S5):376.

20. Sahlin K. Muscle glucose metabolism during exercise. Ann Med. 1990;22(3):85-89.

21. Brooks GA. Importance of the crossover concept in exercise metabolism. Clin Exp Pharmacol Physiol. 1997;24(11):889-895.

22. Wahren J, Ekberg K. Splanchnic regulation of glucose production. Annu Rev Nutr. 2007;27:329-345.

23. Richter EA, Hargreaves M. Exercise, GLUT4 and skeletal muscle glucose uptake. Physiol Rev. 2013;93(3):993-1017.

24. Cooper DM, Barstow TJ, Bergner A, et al. Blood glucose turnover during high and low intensity exercise. Am J Physiol. 1989;257(3 pt 1):E405E412.

25. Greenhaff PL, Timmons JA. Interaction between aerobic and anaerobic metabolism during intense muscle contraction. Exerc sport Sci Rev. 1998;26:1-30.

26. Margaria R, Cerretelli P, Aghemo P, et al. Energy cost of running. J Appl Physiol. 1993;18(2):367-370.

27. Boobis L, Williams C, Wooton SA. Human muscle metabolism during brief maximal exercise. J Physiol. 1982;338:21-22.

28. Quistorff B, Johansen L, Sahlin K. Absence of phosphocreatine resynthesis in human calf muscle during ischemic recovery. Biochem J. 1993;291(Pt 3):681-686.

29. Wasserman DH, Williams PE, Lacy DB, et al. Importance of intrahepatic mechanisms to gluconeogenesis from alanine during exercise and recovery. Am J Physiol. 1988;254(4 Pt 1):E518-E525.

30. Sonne B, Galbo H. Carbohydrate metabolism during and after exercise in rats: Studies with radioglucose. J Appl Physiol. 1985;59(5):1627-1639.

31. Cortright RN, Dohm GL. Mechanisms by Which Insulin and Muscle Contraction Stimulate Glucose Transport Can. J Appl Physiol. 1997;22(6):519-530.

32. Friedlander AL, Casazza GA, Horning MA, et al. Training induced alterations of glucose flux in men. J Appl Physiol. 1997;82(4):1360-1369.

33. Swanwick E, Matthews M. The effects of pre-exercise blood glucose on responses to short duration high intensity exercise. Unpublished data International Journal of Sports and Exercise Medicine. USA; 2018.

34. Kjaer M. Hepatic fuel metabolism during exercise. Exercise metabolism. In: Hargreaves M, editor. Human Kinetics, USA; 1995. p. 73-97.

35. Marliss EB, Vranic M. Intense exercise has unique effects on both insulin release and its roles in glucoregulation. Diabetes. 2002;51(S1):S271$\mathrm{S} 283$

36. Brooks GA, Fahey TD, Baldwin KM. Exercise Physiology: Human Bioenergetics and its Applications. McGraw-Hill Companies: USA; 2005.

37. Coggan AR. Plamsa glucosemetabolism during exercise: effect of endurance training in humans. Med Sci Sports Exerc. 1997;29(5):620-627.

38. Cooper DM, Wasserman DH, Vranic M, et al. Glucose turnover during high and low FIO2 breathing in man. Am J Physiol. 1986;251(2 pt 1):E209-E214.
39. Coggan AR, Raguso CA, Gastaldelli A, et al. Regulation of glucose production during exercise at $80 \%$ of $\mathrm{VO}_{2}$ peak in untrained humans. $\mathrm{Am}$ J Physiol. 1997;273(2 pt 1):E348-E354.

40. Ponsot E, Zoll J, N'guessan B, et al. Mitochondrial tissue specificity of substrates utilization in rat cardiac and skeletal muscles. $J$ Cell Physiol. 2005;203(3):479-486.

41. Gerbitz KD, Gempel K, Brdiczka D. Mitochondria and diabetes: genetic, biochemical, and clinical implications of the cellular energy circuit. Diabetes. 1996;45(2):113-126.

42. Blanchaer MC, Wijhe VM, Mozersky D. The oxidation of lactate and $\alpha$-glycerophosphate by red and white skeletal muscle: I. Quantitative studies. Journal of Histochemistry \& Cytochemistry. 1963;11(4):500-504.

43. Donald MJ, Brown LJ. Calcium activation of mitochondrial glycerol phosphate dehydrogenase restudied. Archives of biochemistry and biophysics. 1996;326(1):79-84.

44. Donald MJ, Marshall LK. Mouse lacking NAD+-linked glycero phosphate dehydrogenase has normal pancreatic beta cell function but abnormal metabolite pattern in skeletal muscle. Arch Biochem Biophys. 2000;384(1):143-153.

45. Contreras L, Puertas GP, Iijima M, et al. Ca2+ Activation Kinetics of the Two Aspartate-Glutamate Mitochondrial Carriers, Aralar and Citrin Role in the Heart Malate-Aspartate Nadh Shuttle. J Biol Chem. 2007;282(10):7098-7106.

46. Easlon E, Tsang F, Skinner C, et al. The malate-aspartate NADH shuttle components are novel metabolic longevity regulators required for calorie restriction-mediated life span extension in yeast. Genes Dev. 2008;22(7):931-944.

47. Gellerich FN, Gizatullina Z, Trumbekaite S, et al. Cytosolic Ca2+ regulates the energization of isolated brain mitochondria by formation of pyruvate through the malate-aspartate shuttle. Biochem $J$ 2012;443(3):747-755.

48. Werth JL, Thayer SA. Mitochondria buffer physiological calcium loads in cultured rat dorsal root ganglion neurons. $J$ Neurosci. 1994;14(1):348-356

49. Wrogemann K, Pena SD. mitochondrial calcium overload: a general mechanism for cell-necrosis in muscle diseases. Lancet. 1976;1(7961):672-684

50. Barron, John T, Liping G, Parrillo JE. Malate-aspartate shuttle, cytoplasmic NADH redox potential and energetics in vascular smooth muscle. J Mol Cell Cardiol. 1998;30(8):1571-1579.

51. Schantz PG, Sjöberg B, Svedenhag J. Malate-aspartate and alphaglycerophosphate shuttle enzyme levels in human skeletal muscle: methodological considerations and effect of endurance training. Acta Physiol Scand. 1986;128(3):397-407.

52. Kane DA. Lactate oxidation at the mitochondria: a lactate-malateaspartate shuttle at work. Front Neurosci. 2014;8:366.

53. Hansford RG, Cohen L. Relative importance of pyruvate dehydrogenase inter-conversion and feedback inhibition in the effect of fatty acids on pyruvate oxidation by rat heart mitochondria. Arch Biochem Biophys. 1978;191(1):65-81.

54. Stanley WC, Wisneski JA, Gertz EW, et al. Glucose and lactate interrelations during moderate intensity exercise in humans. Metabolism. 1988;37(9):850-858.

55. Wilmore JH, Costill DL, Kenney WL. Physiology of Sport and Exercise. Human Kinetics, USA; 2008.

56. Fox EL, Bowers RW, Foss ML. The physiological basis for exercise and sport, 5th ed. Brown \& Benchmark, USA; 1993. 
57. Ernest W. Training Fast Twitch Muscle Fibers: Why And How J Swimming Research. 2012;19:1.

58. Wilmore JH, Costill DL. Physiology of Sport and Exercise. Human Kinetics, USA; 1999.

59. Stainsby WN, Brechue WF, Drobinak ODM, et al. Oxidation / reduction state of cytochrome oxidase during repetitive contractions. $J \mathrm{Appl}$ Physiol. 1989;67(5):2158-2162.

60. Putman CT, Jones NL, Lands LC, et al. Skeletal muscle pyruvate dehydrogenase activity during maximal exercise in humans. $\mathrm{Am} J$ Physiol. 1995;269(3 pt 1):E458-E468.

61. Linnarsson D, Karlsson J, Fagraeus L, et al. Muscle metabolism and oxygen deficit during exercise with hypoxia and hyperoxia. $\mathrm{J} \mathrm{Appl}$ Physiol. 1974;36(4):399-402.

62. Williamson JW, Raven PB, Whipp BJ. Unaltered oxygen uptake kinetics at exercise onset with lower body positive pressure in humans. Exp Physiol. 1996;81(4):695-705.

63. Timmons JA, Gustafsson T, Sundberg CJ, et al. Substrate availability limits human skeletal muscle oxidative ATP regulation at the onset of exercise. J Clin Invest. 1999;101(1):79-85.

64. Amelink GJ, Kamp HH, Bär PR. Heart, Circulation, Respiration and Blood. Environmental and Exercise Physiology. 1988;4.
65. Crowley MA, Willis WT, Matt KS, et al. A reduced lactate mass explains much of the glycogen sparing associated with training. J Appl Physiol. 1996;81(1):362-367.

66. Galbo H. Hormonal and metabolic adaptation to exercise. Stuttgart, Germany, Beorg, thieme, Velag, USA; 1983. p. 112.

67. Hargreaves M, Proietto J. Glucose kinetics during exercise in trained men. Acta Physiol Scand. 1994;150(2):221-225.

68. Hargreaves M. Interactions between muscle glycogen and blood glucose during exercise. Exerc Sport Sci Rev. 1997;25:21-39.

69. Noue LKF, Williamson JR. Interrelationships between malateaspartate shuttle and citric acid cycle in rat heart mitochondria. Metabolism. 1971;20(2):119-140.

70. Mendenhall LA, Swanson SC, Habash DL, et al. Ten days of exercise training reduces glucose production and utilization during moderate intensity exercise. Am J Physiol. 1994;266(1 pt 1):E136-E143.

71. Nimmo GA, Cohen P. The regulation of glycogen metabolism. European Journal of Biochemistry. 1978;87(2):341-351.

72. Noel RJ, Antinozzi PA, Garry JD, et al. Engineering of glycerolstimulated insulin secretion in islet beta cells. Differential metabolic fates of glucose and glycerol provide insight into mechanisms of stimulussecretion coupling. J Biol Chem. 1997;272(30):18621-18627. 